\title{
Una búsqueda de nuevas praxis en torno al uso de las tecnologías de la información y la comunicación: Más allá del ba-be-bi-bo-bu digital
}

A search of new practices around the use of Information and Communication Technologies:

Beyond the digital ba-be-bi-bo-bu

\author{
Volumen 19, Número 1 \\ Sección Especial \\ Enero-Abril \\ pp. 1-10
}

Este número se publica el 1 de enero de 2019

DOI: 10.15517/aie.v19i1.35571

Maricris Castillo Hellmund

Revista indizada en REDALYC, $\underline{\text { SCIELO }}$

Revista distribuida en las bases de datos:

LATINDEX, DOAJ, REDIB, IRESIE, CLASE, DIALNET, SHERPA/ROMEO, QUALIS-CAPES, MIAR

Revista registrada en los directorios:

ULRICH'S, REDIE, RINACE, OEI, MAESTROTECA, PREAL, CLACSO 


\title{
Una búsqueda de nuevas praxis en torno al uso de las tecnologías de la información y la comunicación: Más allá del ba-be-bi-bo-bu digital
}

A search of new practices around the use of Information and Communication Technologies: Beyond the digital ba-be-bi-bo-bu

\section{Maricris Castillo Hellmund ${ }^{1}$}

Resumen: El presente ensayo trata sobre la búsqueda de nuevas praxis en torno al uso de las Tecnologías de la Información y la Comunicación (TIC), acciones que despierten la curiosidad y la sensibilidad, y al mismo tiempo, nos provoquen como seres humanos y no como meras personas usuarias de los recursos tecnológicos digitales. La reflexión surge a partir de una experiencia formativa en la que participaron, protagónicamente, niñas, niños y adolescentes de cinco distritos de la provincia de San José, Costa Rica. El proceso se llevó a cabo generando espacios para la promoción de la cultura tecnológica. Entre sus principales resultados destaca el uso crítico, significativo y empoderador de las tecnologías que hicieron las personas participantes en sus comunidades. De su puesta en marcha se concluye que es fundamental promover iniciativas de alfabetización digital desde perspectivas críticas, las cuales siempre aspiren a ser prácticas de libertad, de diálogo y de solidaridad. El ensayo se compone de tres apartados: en primera instancia, una breve introducción al tema y al contexto en el que se desarrolló la iniciativa. En segundo lugar, la narración y el análisis del proceso formativo, y, por último, se presentan las principales conclusiones.

Palabras clave: alfabetización digital crítica, tecnología de la información, comunicación, jóvenes.

\begin{abstract}
The current paper is about the search for new practices around the use of Information and Communication Technologies (ICT), actions that promote curiosity and awareness and, at the same time, provoke us as human beings and not only as users of the digital resources. The manuscript exposes an education project with the participation of children and adolescents from five districts of San Jose, Costa Rica. The project generated spaces for the promotion of technology culture. Among its main results, it is important to highlight the critical, meaningful and empowering use of technologies did by the participants in their communities. From its implementation, it is concluded that it is fundamental to promote digital literacy initiatives from critical perspectives, which always aspire to be practices of freedom, dialogue and solidarity. The paper has three sections: first, a brief introduction to the topic and the context where the initiative was led. Second, the presentation of the project, and finally, the main conclusions.
\end{abstract}

Key words: critical digital literacy, information technology, communication, adolescents.

\footnotetext{
1 Consultora en temas de educación y protección de derechos de la niñez y la adolescencia. Ha laborado para la UNICEF, Ministerio de Eudcación Pública de Costa Rica, Universidad de Costa Rica, Unión Europea y distintas ONG's como Fundación Paniamor y Acción Joven, Costa Rica.
}

Dirección electrónica: crismari20@gmail.com

Documento recibido: 9 de julio, 2018

Enviado a corrección: 3 de octubre, 2019

Aprobado: 29 de octubre, 2018 


\section{Introducción: ¿Conectad@s?²}

Los selfis, los podcasts, ${ }^{3}$ los celulares, las conexiones a Wi-Fi, los ordenadores, las bases de datos, las redes sociales, las fotografías digitales, entre un extenso etcétera de "cosas", están a nuestro alrededor. La computadora es un mueble más en nuestros hogares, y el celular, pensando en sus diferentes funcionalidades, se ha convertido en una de las prendas más importantes al vestir. Difícilmente salimos de nuestros hogares sin llevarlo en nuestros bolsos o bolsillos; y es poco usual, un día que no entremos a Facebook o no chateemos por WhatsApp. Pareciera que hemos incorporado con bastante normalidad estas tecnologías a nuestras vidas cotidianas, están en nuestras casas, en las calles, están en nuestro imaginario (Bebea, 2015, p. 9).

Por supuesto, sería muy ingenuo plantear que esta es una realidad generalizada, pues la experiencia muestra que el acceso a la información y al conocimiento, a través de herramientas digitales, no está al alcance de todas y de todos, como en ocasiones se llega a suponer. En el caso de Internet, existen en circulación diversas noticias afirmando que más de 4000 millones de personas alrededor del mundo (más de la mitad de la población del planeta) no tienen acceso a este servicio (Banco Mundial, 2016).

Por su parte, la base de datos de Indicadores de desarrollo mundial señala que, en Costa Rica para el 2015, las personas usuarias de la red correspondían a un $59.8 \%$ de la población (Banco Mundial, 2018). Cifra digna de ser analizada cuando se consideran los cientos de centros educativos en la zona Norte sin señal de teléfono y sin internet (Miranda, 2017)4; o cuando el Quinto Informe del Estado de la Educación (2015) menciona: "el porcentaje de hogares con acceso a internet en los distritos rurales pobres, rurales no pobres y urbanos es del $8 \%, 15 \%$ y $37 \%$ respectivamente" (p. 269).

Ahora bien, aunque en los últimos años el acceso a las Tecnologías de la Información y la Comunicación (en adelante TIC) ha crecido "rápidamente", no se puede olvidar que aún hay un número significativo de personas con condiciones muy distintas. Amador Zamora (2016), investigador del Programa Sociedad de la Información y Conocimiento (PROSIC) de la Universidad de Costa Rica (UCR), dice:

\footnotetext{
2 Adrede, se coloca un “@” en lugar de las vocales "a” y "o", con la intención de hacer referencia a la vida digital.

${ }^{3}$ Respectivamente, auto-fotografías y archivos de audio gratuitos.

${ }^{4}$ Artículo publicado en el Semanario Universidad.
} Volumen 19 Número 1 (Enero-Abril), ISSN 1409-4703 
Uno de los temas que debe tener mayor relevancia en las aspiraciones nacionales es la brecha digital multidimensional: los hogares de zonas rurales, de escasos ingresos y de bajo nivel educativo, no tienen la misma oportunidad de acceso que el resto del país [...]. (p. 151)

Entonces, si se parte de que el acceso a la información y la libertad de expresión son derechos fundamentales, ¿qué sucede cuando hay personas excluidas de estos espacios en donde se producen nuevas formas de socialización?, ¿qué pasa cuando no se puede pagar la recarga para tener acceso a los servicios de telefonía e internet?, o ¿qué ocurre cuando hay niñas y niños que apenas saben usar un mouse ${ }^{5}$, cuando ya hay otros que grafican complejas funciones matemáticas en sus tabletas escolares? Planteo estas preguntas, primero, porque haber nacido y crecido con cierto tipo de tecnologías ya instaladas en la sociedad no es sinónimo de tener acceso a ellas (Ferreiro, 2011). Segundo, porque fue en este contexto y con estas condiciones que participé en una experiencia ${ }^{6}$ (un proceso formativo con personas jóvenes), la cual comparto a través del presente ensayo.

Grosso modo, el Club Innovatorio es una iniciativa impulsada por la Organización No Gubernamental (ONG) costarricense, Fundación Paniamor, que ha buscado generar espacios para la promoción de la cultura tecnológica en niñas, niños y adolescentes, entre los 10 y los 18 años. Para julio del año 2016 y junio de 2017, periodo que abarca este documento, el proyecto formativo se desarrolló en cinco distritos de la provincia de San José. Participaron personas jóvenes, quienes contaron con la colaboración de otras actrices y actores comunitarios, tales como familiares, asociaciones de desarrollo, iglesias, bibliotecas y dos centros educativos. En cuanto a la propuesta formativa, la visión del reconocido pedagogo brasileño Paulo Freire, referente en procesos de alfabetización y cuyo pensamiento resulta más actual que nunca, resultó una herramienta clave. Él decía que "la educación verdadera es praxis, reflexión y acción del hombre sobre el mundo para transformarlo" (Freire, 1969, p. 7).

Finalmente, agradezco a la Fundación por su apertura para compartir esta experiencia y reflexionarla críticamente. A su vez, aclaro que este ensayo no refleja, necesariamente, su posición.

${ }^{5}$ Dispositivo apuntador que permite interactuar con una computadora. (http://definicion.de/mouse/, 2017)

${ }^{6}$ El rol de la autora de este ensayo en la iniciativa correspondió a la coordinación metodológica. Volumen 19 Número 1 (Enero-Abril), ISSN 1409-4703 


\section{El proceso formativo}

Cuando se plantea un trabajo con personas adolescentes desde una ONG, una universidad, en fin, cualquier organización, es muy importante no caer en una concepción ingenua de la alfabetización. Con ello, Freire (1969) apunta a que no se puede perder de vista que los procesos de alfabetización no son regalos de los que "saben", para los que "no saben" (p.12). No son caridad ejercida desde una posición vertical, retomando las palabras de Galeano.

En relación con este tema, me atrevo a sostener la hipótesis de que los procesos formativos vinculados con las TIC y con las personas jóvenes tienen una pequeña/gran ventaja que puede servir para hackear las posturas de poder/saber ${ }^{7}$ asumidas, en ocasiones, por quienes educan. ¿Y cuál es esta particularidad? Justamente que niñas, niños y adolescentes suelen tener conocimiento sobre las TIC y si aún no tienen apropiación sobre ellas, es un tema que por lo general les interesa, un asunto que no es ajeno a su cultura juvenil y sobre el cual construyen conocimiento muy rápidamente (Ferreiro, 2011). Entonces para las personas educadoras no se vuelve tan sencillo caer en esa trampa de "transmisión mecánica de conocimientos", de "memorícese este ba- be- bi- bo- bu digital", porque en repetidas ocasiones es la población infantil/juvenil la que explica cómo utilizar un app o una tableta, cómo hacer un video mediante un nuevo programa, cuáles son las redes sociales que están de moda, o cómo se comparte internet desde el celular. Esto no quiere decir que las personas adultas no sepan o no puedan, solamente que es significativo resaltar el dominio, el saber, el atrevimiento y el disfrute que pueden desarrollar las personas más jóvenes con estas, también llamadas, nuevas tecnologías. Ferreiro (2011, p. 425) expresaba que "no es lo mismo ver llegar una tecnología, que nacer con ella ya instalada en la sociedad". D’Antoni (2012), por su parte, decía que: "Los medios digitales proporcionan, como es sabido, un mundo de información y de diversión que tiene características como el atractivo, la velocidad, los cambios continuos [...]" (p. 48).

Conscientes de este interés y frecuente dominio de las nuevas generaciones sobre las TIC, nosotras, como educadoras y como colectivo de trabajo, en lugar de imponer nuestro saber, decidimos aprovechar sus conocimientos. Es decir, formulamos un proyecto en el que las ideas y propuestas de las personas jóvenes en torno al uso de las tecnologías fueran siempre importantes. Así logramos que se realizara un uso crítico, seguro, responsable,

\footnotetext{
${ }^{7}$ En un sentido Foucaultiano (2003).
} Volumen 19 Número 1 (Enero-Abril), ISSN 1409-4703 
significativo y empoderador ${ }^{8}$ de los recursos digitales (Fundación Paniamor, 2017). Asimismo, en lugar de pensar en un proceso formativo meramente instrumental, centrado más en el aprendizaje del uso de las herramientas tecnológicas, tratamos de poner en práctica uno en el que las personas, sus necesidades y sus cotidianidades fueran el eje central y transversal de todo el proyecto.

Para junio del 2017, la iniciativa se había desarrollado en cinco distritos de la provincia de San José: Brasil y Uruca de Santa Ana, Grifo Alto y Santiago de Puriscal, y en el barrio de Sagrada Familia del distrito de Hatillo, en el cantón central de San José. La selección y la participación de estas comunidades se vinculó con diferentes razones. En un principio, con la consideración de información proporcionada por instrumentos como el Índice de Bienestar de la Niñez y la Adolescencia (2014) del Ministerio de Planificación Nacional y Política Económica (MIDEPLAN), las Estadísticas sobre el Acceso a las TIC y la Densidad Poblacional (2011) del Instituto Nacional de Estadísticas y Censos (INEC); además de recomendaciones hechas por el Patronato Nacional de la Infancia (PANI). Luego, y de suma relevancia, con el interés de las comunidades y sus diferentes actrices y actores por participar.

Si bien, el proceso formativo se desarrolló con niñas, niños y adolescentes, una gran parte del proyecto se articuló gracias al apoyo de sus familiares y de la participación activa de fuerzas vivas comunitarias. En Brasil, por ejemplo, un grupo de vecinas y vecinos pertenecientes a la filial de la Iglesia Católica se encargaron de gestionar espacios y electricidad para trabajar. En Grifo Alto, fue la escuela pública de la localidad, y en Sagrada Familia, la biblioteca María Luisa Porras Monge. Freire (1969) lo decía muy bien cuando planteaba: "somos seres de relaciones y no sólo de contactos, no sólo estamos en el mundo sino con el mundo" (p.28). Es en este tejido de lazos sociales en el que, actualmente, se han inscrito 142 participantes entre los 10 y los 18 años. "Actualmente", porque el proyecto está por continuar, y "participantes" porque en los procesos que se piensan desde la Pedagogía Crítica, la gente que participa, nunca es beneficiaria. No se trata de sujetos con mentes vacías que reciben y repiten, se trata de personas que piensan y proponen. Citando a D’Antoni, Gómez Ordoñez, Gómez Torres y Soto Arguedas (2011), "buscando (des) (re) educar a las personas para que pudieran alcanzar una formación integral y no reprodujeran ni legitimaran procesos instruccionales y enceguecedores" (p. 39).

${ }^{8}$ Se puede revisar con mayor detalle la Propuesta de Crianza Tecnológica de la Fundación. En ella se promueve el hacer un uso seguro, responsable, significativo y empoderador de las TIC. 
¿Y qué fue exactamente lo que se hizo en el proceso formativo? Lo imaginamos como un ejercicio práctico en un taller creativo. Propusimos una metodología participativa que articulaba herramientas de la tecnología, la ciencia y el arte. Como expresaba Bebea (2015): "Queríamos liberarnos de la especialización y de los libros de texto, ir más allá de lo que nos contaron primero en la escuela, luego en la universidad y más tarde en un puesto de trabajo como modernos obreros frente a un ordenador". En nuestro caso aceptamos los cambios en el contenido del programa y validamos los errores en el proceso. Apoyamos que las chicas y los chicos propusieran temas, actividades, opinaran siempre sobre lo que les interesaba y lo que no. ¿De qué serviría que aprendieran a usar PowToon ${ }^{9}$ o $\operatorname{Prezi}^{10}$ si no lo encuentran importante en sus vidas cotidianas?, o, ¿cuál sería el fin de una experiencia formativa relacionada con las TIC, si solo nos imaginamos como consumidoras y consumidores, 0 como robots que repiten instrucciones?

En cuanto al tiempo de ejecución, el proceso estuvo conformado por nueve sesiones en los distritos de Santa Ana y de Puriscal, y cuatro en Sagrada Familia. Cada uno de estos encuentros se nombraba de tal manera que orientara el contenido temático. Por ejemplo, la primera sesión recibía el nombre de Tecnologías para el bien: aprender compartiendo. La segunda, Uso significativo de las TIC; la tercera, Nuestra comunidad: pensemos en los retos; la cuarta, Cultivando sueños, empoderando acciones y así sucesivamente.

En ellas, además de experimentar con programas como Canva, Moovly, Coogle, hacer animaciones digitales en Stop Motion ${ }^{11}$, crear robots con motores y baterías, o aprender principios básicos de programación con los Arduinos $^{12}$ se generaron espacios para pensar y dialogar sobre los temas que los grupos considerasen necesarios. Por ejemplo, sobre nuestra posición en el mundo frente a estas nuevas tecnologías y frente a quiénes las manejan, sobre nuestro derecho a la información y a la gestión desde el bien común, sobre el aprovechamiento de las TIC para mejorar las condiciones en las comunidades y sobre la posibilidad de transformarlas en tecnologías para el aprendizaje y el conocimiento (TAC), o

\footnotetext{
${ }^{9}$ Herramienta web que permite crear videos y presentaciones animadas.

10 Herramienta para la creación de presentaciones, similar a Microsoft Office Power Point.

11 Canva es una herramienta para diseñar contenido web, como carteles, iconografías, gráficos, posters, entre otros. Moovly, por su parte, es una herramienta online para crear contenidos multimedia, como videos y presentaciones animadas. Coogle, una herramienta web para crear mapas mentales. Y Stop Motion es una técnica de animación que consiste en simular el movimiento de objetos estáticos por medio de una sucesión de imágenes fotografiadas.

12 Un arduino "es una plataforma de prototipos electrónica de código abierto (open-source) basada en hardware y software flexibles y fáciles de usar. Está pensado para artistas, diseñadores, como hobby y para cualquiera interesado en crear objetos o entornos interactivos". (Arduino.cl, 2017)
}

Volumen 19 Número 1 (Enero-Abril), ISSN 1409-4703 
mejor aún, en tecnologías para el empoderamiento y la participación social (TEP) (Fundación Paniamor, 2017).

El Club Innovatorio generó espacios para que las personas jóvenes pensaran en retos de sus comunidades, y a partir de estas ideas crearan sus propios proyectos utilizando las tecnologías. Barreiro (1969), retomando a Freire, hablaba sobre la importancia de aquellas palabras que encarnan la realidad de quien las pronuncia y no de esas que son vehículos de ideologías alienantes (p.13). Entonces, en lugar de imponerles sobre qué desafío ocuparse, las personas participantes propusieron los retos que les interesaba abordar. Algunos ejemplos de lo que nombraron son: la contaminación en sus barrios, los perros callejeros abandonados, las balaceras, la falta de comprensión en sus familias y la ausencia de espacios para jugar.

Después de este proceso de identificación y análisis, comenzaron a preparar sus proyectos. Trabajaron en grupos, exploraron las herramientas, jugaron, travesearon, ingeniaron, cooperaron, buscaron tutoriales en YouTube cuando no tenían la menor idea de qué hacer o cómo hacerlo $\mathrm{y}$, felizmente, en algunas ocasiones contaron con el acompañamiento de personas pertenecientes a diferentes organizaciones, como la Asociación Centroamericana de Aeronáutica y del Espacio (ACAE), la Casa de la Cultura de Ciudad Colón y la Empresa de Servicios en Energía en las Américas (ESCOIA). Vídeos, páginas en Facebook e Instagram con fines de interés social, robots hechos con basura, canales de YouTube de jóvenes hablándoles a otras y a otros jóvenes, son algunos de los ejemplos de las iniciativas que desarrollaron. Además, y esto se confirmó gracias a unos instrumentos de investigación utilizados ${ }^{13}$, al final del proceso, muchas de las personas participantes se reconocían a sí mismas como personas críticas, creativas, soñadoras y solidarias, capaces de utilizar las tecnologías para algo más. A continuación, algunas de sus respuestas ante la pregunta: “¿Después de haber participado en este proceso para qué te gustaría utilizar la tecnología?":

"Para ayudar a la comunidad, colegio, familiares y hasta mi misma."

"Para crear cosas nuevas."

"Para hacer el mundo mejor."

"Para hacer los proyectos que me gustan."

\footnotetext{
${ }^{13}$ Se utilizaron cuestionarios al principio y al final del proceso, con la intención de conocer el perfil de entrada y de salida de las personas participantes jóvenes.
} Volumen 19 Número 1 (Enero-Abril), ISSN 1409-4703 
"Para realizar trabajos en el cole más creativos."

"Para ayudar a quien lo necesite, y aparte ayudarme a mí misma con mis estudios y carrera a futuro."

En lo que respecta a los alcances y a los límites, el primero de ellos fue el tiempo de realización del proceso formativo. El segundo, la cantidad de espacios disponibles en cada comunidad. Si bien el proyecto hizo siempre un máximo aprovechamiento de los recursos existentes, es significativo considerar estos aspectos porque se han hecho esfuerzos para sostener la iniciativa en un mayor lapso y con la participación de más personas, también en espacios fuera de la Gran Área Metropolitana (GAM). El tercer aspecto es que, aunque el proyecto se formuló con un enfoque inclusivo, es necesario continuar pensando en más estrategias que garanticen la integración digital de las personas con discapacidad. Actualmente, se han puesto en práctica acciones modestas, pero no por ello poco relevantes, como subtitular e introducir sonido a todos los vídeos, o bien, utilizar las funciones de accesibilidad disponibles en los dispositivos utilizados.

Por último, en todas las localidades en donde se realizó el proceso formativo, se llevó a cabo una actividad de apertura y una de cierre. En la primera, se compartió información sobre la iniciativa y se invitó a las personas jóvenes a inscribirse. En la segunda, se buscó vincular aún más a los grupos participantes con sus comunidades, a través de espacios de encuentro con pares y referentes adultos, para la presentación de sus proyectos.

\section{Apreciaciones finales}

En la actualidad, vivimos en lo que se ha llamado Sociedad de la Información o Sociedad del Conocimiento, un momento en el que la humanidad entiende su progreso estrechamente vinculado con los avances de la ciencia y de la tecnología, hasta el punto de considerarlos indicadores de referencia sobre el desarrollo de una sociedad; dadas estas circunstancias, sugiere Bebea (2015), cada vez surgen más y más propuestas que sirven para promover la formación técnica y la especialización de los usuarios. Desde edades tempranas hasta la adultez una gran cantidad de personas "adquieren una educación eficaz en tanto asimilan las destrezas necesarias para manejar las herramientas digitales: la alfabetización digital" (Bebea, 2015, p. 9).

Sin embargo, si deseamos una sociedad libre, necesitamos una educación libre, una que promueva el pensamiento crítico, ético y creativo, y que no niegue ni rechace las 
tecnologías, sino que las acepte "pero no para adaptarnos mansamente a las directrices de la eficacia, sino para incluirnos e implicarnos transformando su uso [...] "(Bebea, 2015, p. 10)

El Club Innovatorio, lejos de querer convertirse en un manual lleno instrucciones, ha representado una búsqueda de nuevas praxis en torno al uso de las TIC, una estrategia "para explorar formas de humanizarnos en un mundo digitalizado" (Bebea, 2015, p.9). Educandos y educadores con educadores y educandos (Barreiro, 1969) ${ }^{14}$ constantemente nos hemos preguntado: ¿para qué estamos utilizando las tecnologías?, ¿realmente nos hacen libres? Hemos partido de que aprender a utilizar las herramientas tecnológicas es importante, pero comprender y transformar la cultura que generamos al usarlas, no solo tiene sentido, sino que es esencial. Parafraseando a Freire, nos entregamos al esfuerzo crítico de trabajar en un proyecto de formación y no de mero entrenamiento técnico-profesional.

En cuanto a los resultados del proceso formativo, podría pensarse que fueron los proyectos elaborados por las personas jóvenes (vídeos, páginas de Facebook e Instagram, Arduinos, entre otros ejemplos) y su respectiva socialización en sus comunidades. Sin embargo, más allá de estos materiales tangibles, lo que resulta realmente importante es el proceso y el espacio que tuvieron los grupos participantes para expresar su voz. De eso se trata, de plasmar ideas, de hacerlo segura y responsablemente en los espacios virtuales para romper con un tipo de lógica adultocéntrica, jerárquica y disciplinaria, que exige "tener" más de 18 años (entre otras cosas) para poder participar. En Brasil, por ejemplo, uno de los grupos fue invitado a exponer su proyecto en una reunión de la Asociación de Desarrollo del distrito. En Santiago, algunas de las personas participantes se reunieron con la vicealcaldesa para hablar de una propuesta en redes sociales que abordaría el tema de los perros callejeros de la localidad.

Asimismo, es necesario continuar sumando esfuerzos que garanticen el acceso para todas las personas y no solamente para algunas. En especial, porque la vida digital es un nuevo campo para el desarrollo de la ciudadanía, un lugar en el que también se pueden ejercer prácticas que nos permitan tener una mayor conciencia para construir un mundo mejor.

\footnotetext{
14 "No más educando, no más educador, sino educador-educando con educando-educador, como el primer paso que debe dar el individuo para su integración en la realidad nacional, tomando conciencia de sus derechos." Palabras de Barreiro retomando el pensamiento de Freire. (Barreiro,1969, p.16). 


\section{Referencias}

Amador Zamora, Alejandro. (2016). Acceso y uso de las TIC en los hogares costarricenses. En PROSIC, Informe hacia la Sociedad de la Información y el Conocimiento 2016 (pp. 151-186). Recuperado de http://www.prosic.ucr.ac.cr/sites/default/files/recursos/cap4 2016.pdf

Arduino.cl. (2017). ¿Qué es arduino? Recuperado de http://arduino.cl/que-es-arduino/

Banco Mundial. (2016). Tecnologías digitales: su enorme potencial de desarrollo aún escapa a los 4000 millones de personas que no tienen acceso a internet. Recuperado de http://www.bancomundial.org/es/news/press-release/2016/01/13/digital-technologieshuge-development-potential-remains-out-of-sight-for-the-four-billion-who-lack-internetaccess

Banco Mundial. (2018). Indicadores del desarrollo mundial. Recuperado de http://databank.bancomundial.org/data/source/world-development-indicators\#

Barreiro, Julio. (1969). Educación y concienciación. En Paulo Freire, La Educación como práctica de libertad (pp. 7-19). Montevideo, Uruguay: Siglo XXI Editores.

Bebea, Inés. (2015). Alfabetización Digital Crítica. Una invitación a reflexionar y actuar. Recuperado de http://ondula.org/publicaciones/

D’Antoni, Maurizia. (2012). Interés por la Pedagogía Crítica: ideas sobre unas articulaciones. Revista Ensayos Pedagógicos, 7(1), 33-53. Recuperado de http://www.revistas.una.ac.cr/index.php/ensayospedagogicos/article/view/6018

D’Antoni, Maurizia, Gómez Ordoñez, Luis, Gómez Torres, Juan y Soto Arguedas, José Fabio. (2011). La desobediencia como forma de resistencia: jóvenes que se hacen sujetos en contextos de "declive" institucional. Al respecto de la enseñanza colegial costarricense. Revista Ensayos Pedagógicos, 6(1), 37-54. Recuperado de http://www.revistas.una.ac.cr/index.php/ensayospedagogicos/article/view/4471

Estado de la Educación. (2015). Quinto Informe Estado de la Educación 2015. Recuperado de http://www.estadonacion.or.cr/educacion2015/

Ferreiro, Emilia. (2011). Alfabetización Digital. ¿De qué estamos hablando? Revista Educação e Pesquisa, 37(2), 423-438. doi: http://dx.doi.org/10.1590/S151797022011000200014

Foucault, Michel. (2013). El Poder, una bestia magnífica. México: Siglo Veintiuno Editores.

Freire, Paulo. (1969). La Educación como práctica de libertad. Montevideo, Uruguay: Siglo XXI Editores.

Fundación Paniamor, Costa Rica. (2017). Crianza Tecnológica, Viviendo la Ciudadanía Digital. Recuperado de http://www.crianzatecnologica.org

Miranda, Hulda. (27 de junio de 2017). Fonatel falló en llevar a Internet a estudiantes de zona Norte. Desconectados del Presente. Semanario Universidad. Recuperado de https://semanariouniversidad.com/pais/desconectados-del-presente/ 